\title{
Relational perception and modes of perceiver operation '
}

\author{
HAROLD W. HAKE, GERALD W. FAUST, JOHN S. MCINTYFE AND HARRY G. MURRAY
}

THE UNIVERSITY OF ILLINOIS

Judgments were made of the size of a small, luminous (inner) square appearing in the center of a somewhat larger, variable (outer) square, the whole pattern appearing in the dark. Accuracy of judgment was greatest when the outer square was constant from trial to trial but deteriorated when the outer square also varied in size, reaching a lower limit less than the accuracy produced under the absolute judgment condition (no outer square presented). An application of multiple discriminant analys is and the use of a maximum likelihood observer model provided estimates of the extent to which Os responded to inner and outer size variation as separate aspects of stimulation. Although Os differed markedly in this respect, the ir ability to identify inner square size under the various conditions did not reflect this difference.

The relational properties of visual stimulation, emphasized as a principle of perception in the Gestalt tradition, have been described empirically by a class of research studies. Estimates of the size of a simple figure have been shown to be affected by the size or shape of the surround or context in which the figure is presented. For example, the percelved length of a vertical line centered within a rectangle depends upon rectangle size as well as upon real line length (Künnapas, 1955; Rock \& Ebenholtz, 1959). The same type of contextually-produced constant errors in the estimation of the size of a circle within a circle, a square within a square, and in a variety of other configurations was reported by Obonai (1954).

These reports imply a particular result in the experimentation reported here. Observers were required to judge the size of a small luminous square appearing in the center of a somewhat larger luminous square, the whole pattern occurring in the dark. From trial to trial both the outer square and the inner square varied in size, and over all trials all combinations of inner and outer square sizes appeared equally often. The result implied by the relational studies is that judgment of the size of the inner square should be determined by outer square size as well as by inner square size. In that event, accuracy of judgment of inner square size over many trials would be limited. Outer square size would be a source of noise perturbing the source of signal (inner square size variation).

The usefulness of communication models in this application was suggested earlier (Hake, Rodwan, \& Weintraub, 1966). After considering possible modes of perceiver operation, it was suggested that perceptual performance can be represented best as a mixed case. This is an optimum case that lies between extreme alternatives. These have been described by Uhr (1963), in terms of computer models, as "template matching" and "primitive input analysis." In the first model, recognition and identification of aspects of input are accomplished by operations that match aspects of input with stored, absolute recognition standards. Such a system could permit accurate absolute judgment of stimulation, provided the required templates are stored and maintained in precise calibration with all possible input states. The second alternative model responds only to relations existing within complex inputs. It avoids the difficulties associated with the storage and calibration of innumerable absolute standards of recognition but lacks ability to respond absolutely to single aspects of complex inputs. In the case of the stimuli of this study the "analytic model" would predict considerably better accuracy in the judgment of the size of the inner square when presented in the center of an outer square of constant size than when presented alone. When the inner square is seen in the context of the outer square a standard of recognition, the size of the constant outer square, exists in immediate input and can be used in the judgment of inner square size. The relationship between the variable inner square size and the constant outer square size would be crucial to a perceiver described by the analytic model, but would be irrelevant to a perceiver who used stored, implicit recognition standards applied to inner square size alone. The two models, then, may be characterized in terms of the extent to which they depend upon external recognition standards occurring in stimulus inputs.

The mixed model suggested by Hake et al supposes a system that depends upon very crude, unstable, internal standards of recognition that can be sharpened, stabilized, or replaced by relatively constant contextual aspects of immediate inputs. The greater the gain in accuracy possible in this way when stimuli include aspects that remain relatively constant over time, the greater must be the failure of the system when the judged aspect and other contextual aspects both vary and do so in an uncorrelated fashion. This supposes that the mixed model represents a stable mode of operation. That is, the observer (O) is not able to change markedly, depending upon the conditions of observation, the extent to which external, contextual standards of recognition are necessary for accurate judgment.

The stability of the mode of operation of Os in 


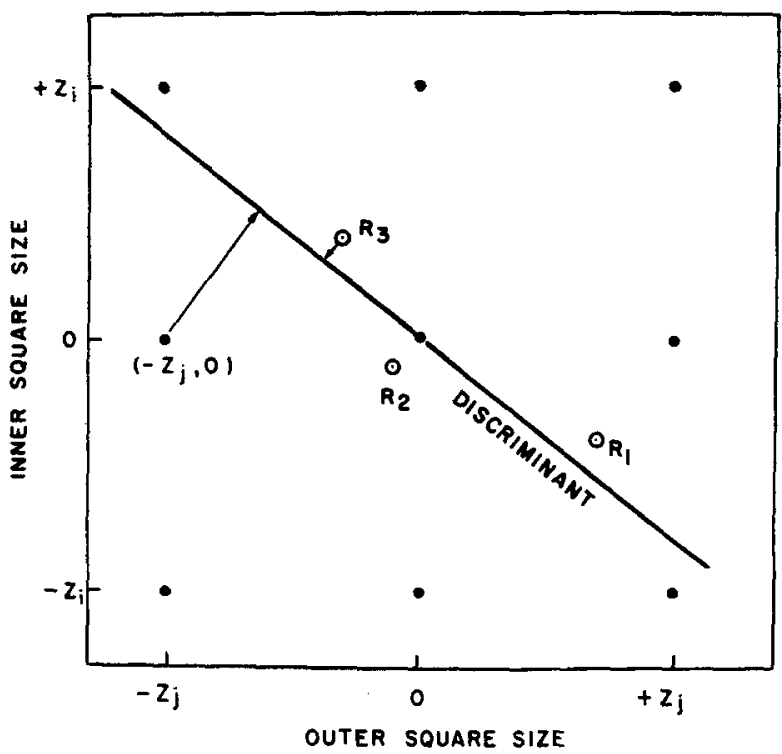

Fig. 1. The stimuli (solid circles) and response class centroids (open circles) are represented as points in the stimulus plane. Both inner square size and outer square size are indicated in units of their respective standard deviations. The reflections of a stimulus point and a centroid point upon the judgmental axis (discriminant) are illustrated.

this experimentation was tested by varying the range of sizes of the outer square appearing in a series of trials. Os were required to classify the size of the inner square when presented alone (Absolute condition), when presented in the center of an outer square of constant size (Constant condition), and when combined with one of three ranges of size variation of the outer square (Orthogonal conditions). The supposition was that if the mode of perceiver operation remained constant over the conditions, then judgmental accuracy should be better in the Constant than in the Absolute condition and should decline progressively with increasing range of variation of the outer square in the Orthogonal conditions. In fact, if the mode is fixed as a perceiver characteristic, then some range of variation of the outer square must produce an accuracy of judgment of the inner square inferior to that produced in the Absolute condition.

An analytic method was applied to describe the mode of operation in greater detail under the Orthogonal conditions. The objective was the precise determination of the extent to which the judgments of Os in these conditions could be understood as a complete failure to discriminate between outer square size and inner square size as separate aspects of stimulus variation from trial to trial. An extreme analytic perceiver model, the "non-discriminating" model, assumed that some linear combination of inner and outer square sizes determined entirely what was perceived and judged. This supposition is illustrated in Fig. 1, which portrays the nine stimulus combinations of inner and outer square size in a stimulus plane. Although inner square size variation was seldom equal to outer square size variation, the stimulus plane is pictured as a square because inner and outer square sizes are plotted in units of their respective standard deviations computed over all trials of a condition. If judgments of an $O$ are determined solely by a linear combination of inner and outer square size, then the diagonal line in the figure, the discriminant, could represent this combination. It represents the direction of stimulus variation that $O$ accepts as being variation in inner square size alone, stimulus variation in any other direction in the plane being not sensed.

An equivalent assumption is that the stimuli may be adequately represented by their reflected positions on the discriminant, as indicated for one stimulus in the figure, when the discriminant is a line drawn arbitrarily through the intersect. In what follows the discriminant is referred to as a line in this position, with the stimuli represented by their positions reflected on it. Since all stimuli were presented equally often, this interpretation of the discriminant is equivalent to its definition as a direction in the stimulus space. In either case the assumption that the twodimensional stimuli were perceived unidimensionally means that the stimuli can be adequately represented by variation in a single direction in the stimulus plane, that direction being indicated by the discriminant line. No other direction of variation matters.

Under that assumption, the orientation of the discriminant line in the Orthogonal conditions, as indicated by $O ' s$ judgments of inner square size, should depend only upon the range of variation of the outer square. This must follow from the fact that inner square variation is a constant in all conditions and from the supposition that os perceive only the linear combination of inner and outer square size.

Three points in Fig. 1 represent response centroids, the $R_{k}$. The coordinates of each are the mean of all the inner square sizes and the mean of all the outer square sizes identified by that response. The simple non-discriminating model implies that these centroids should lie very close to the line representing the combination of stimulus aspects being judged. Hence, the projections of these centroids on this line should be separated more than are their projections on the vertical axis representing inner square variation alone. And, the extent to which the centroid projections on the judgmental axis are separated should be an increasing function of the range of variation of the outer square sizes. Since $O$ judges a combination of inner and outer square size, greater variation in outer square size increases the total range of the stimulus combination being judged.

These implications of the non-discriminating model were tested by an application of multiple discriminant analysis that yielded a quantitative description of the discriminant for each Orthogonal condition. The extent to which variation among the response centroids 
corresponded to the direction of the discriminant could then be determined. In addition, the application of a maximum likelihood observer model provided a measure of the extent to which the judgments of each stimulus could be predicted entirely from stimuius reflections on the discriminant. The accuracy of these predictions was compared with predictions made under the alternative hypothesis that os could discriminate between inner and outer square sizes.

\section{Observers}

\section{METHOD}

The three paid Os were graduate students in psychology. They were given extensive practice in the judgment of square size in the apparatus prior to experimental procedures and were fully informed about each experimental condition prior to attempting it.

\section{Apparatus}

On each trial the $O$ placed his face in a viewing hood and looked through an 8-ft, dark viewing tunnel into a tachistoscope (Gerbrands, Mociel T-2B). In each channel of the tachistoscope a milk glass screen was backlighted by a single fluorescent tube. The luminance of each screen was .13 ft-L. Directly in front of the screen in each channel was a system of vertical and horizontal sliding doors that could be adjusted to create a square opening the center of which was always in the same position. Positions of these doors were continuously adjustable. Prior to each condition the beam splitter in the tachistoscope was checked and adjusted to insure that small squares produced in one channel were always exactly centered in the larger square openings produced in the other channel. The smaller, inner squares appeared as luminous squares centered within larger squares of somewhat less luminance. The fluorescent tubes (Sylvania $4 \mathrm{~W}$ Daylight) were energized by a standard power supply (Gerbrands, Model T-2B), and the duration of stimulus appearances was controlled by a system of electronic timers (Hunter, Model 111-C).

\section{Procedure}

Prior to each trial the $O$ kept his head away from the viewing hood to prevent progressive dark adaptation and viewed a lighted wall (23 ft-L) at a distance approximately equal to the viewing distance of the apparatus (10 ft). At a signal from $E$, O placed his head against the hood and triggered the trial sequence. After the trial he reported verbally his judgment of inner square size, recorded it, and looked back at the wall. The O's judgment alternatives were that the inner square seen was the small $(34 \mathrm{~mm})$, medium (35 $\mathrm{mm})$, or large $(36 \mathrm{~mm})$ size. These judgments were recorded by $O$ as the numbers 1,2 , or 3 , respectively.

The $O$ was not informed of the accuracy of his response on each trial. The objective was not to learn the limits of what he could be taught to do, but rather to learn about his solutions to the problems posed by the conditions. In the Orthogonal and Constant conditions a trial consisted of a 1-sec appearance of the outer square alone followed immediately by a 1-sec appearance of the inner square and the outer square together. In the Absolute conditions a trial consisted of a 1-sec appearance of a small, dim fixation cross followed by a $1-\mathrm{sec}$ appearance of the inner square alone. The Constant condition was the same as the Orthogonal conditions except that outer square size did not vary from trial to trial. The inner square size on a trial was 34,35 , or $36 \mathrm{~mm}$ on a side, the three sizes appearing equally often within a random trial series. The sizes of the outer squares used in the various conditions are shown in Table 1. These sizes also appeared equally often in a Series and formed an orthogonal set with inner square size within each random Series.

The 270 trials in each condition were run in series of 54 trials each. In a single session, five series were run, one series of trials in each condition. The order of the series in each of five sessions for each $O$ was determined by the rows of a 5 by 5 Latin square. Prior to each condition the $O$ was given 18 practice trials, two of each stimulus combination in the Orthogonal conditions. In all cases six practice trials involved each inner square size. After all sessions had been run for each $O$, trials in the five series of each condition were combined.

\section{RESULTS}

The responses recorded over the 270 trials of each Orthogonal condition formed three response tables. As shown in Table 2, each response table consisted of nine cells determined by three rows (inner square size, $s_{i}$ ) and three columns (outer square size, $S_{j}$ ). Each cell in a table contained the frequency, $f_{j j k}$, with which that combination of inner and outer square size was classed in response class $\mathbf{R}_{\mathbf{k}}$. In the Absolute and Constant conditions the three response tables each consisted of a single column of cells. A cell in response class $R_{k}$ contained a frequency of $f_{i . k}$ with which that inner square size was classed in response class $R_{k}$.

Table 1. Outer Square Sizes in the Five Conditions

\begin{tabular}{|c|c|c|c|c|c|}
\hline \multirow{3}{*}{$\begin{array}{l}\text { Square } \\
\text { Size }\end{array}$} & \multicolumn{5}{|c|}{ Conditions } \\
\hline & \multirow[t]{2}{*}{ Absolute } & \multirow[t]{2}{*}{ Constant } & \multicolumn{3}{|c|}{$\begin{array}{l}\text { Orthogonal Condition Rarige } \\
\text { (in mm.) }\end{array}$} \\
\hline & & & 2 & 4 & 6 \\
\hline $47 \mathrm{~mm}$ & & & & & $x$ \\
\hline 48 & & & & $x$ & \\
\hline 49 & & & $x$ & & \\
\hline 50 & & $x$ & $x$ & $x$ & $x$ \\
\hline 51 & & & $x$ & & \\
\hline 52 & & & & $x$ & \\
\hline 53 & & & & & $x$ \\
\hline
\end{tabular}


Table 2. The Response Tables

(Rows refer to inner square size, $s_{i}$, and columns refer $t r$ ater square size, $s_{\mathbf{j}}$ )

$$
R_{1}: \text { "Small"" }
$$
$\left(S_{j}\right)$

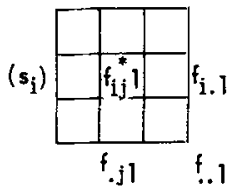

$\left(\mathbf{s}_{\mathbf{i}}\right)$

$R_{2}:$ "Medium"

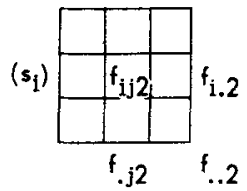

$\mathrm{R}_{2}$ : "Large" $\left(S_{j}\right)$

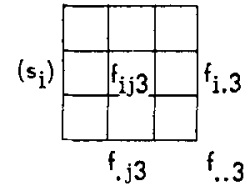

* The frequency of classification of stimulus $\left(s_{i} s_{j}\right)$ in response class $R_{1}$.

\section{Accuracy of Response to Inner Square Size}

The measure of the accuracy of an $O^{\prime} \mathrm{s}$ classification of inner square size was the ratio, $B / W$, of the between-response-class sum of squares to the within-response-class sum of squares. It was chosen because of its generality. It is interpretable in the same way as subsequent, identical measures of more complex performance where accuracy, in the sense of veridicality, cannot be strictly defined.

For the Absolute and Constant conditions the between-response-class sum of squares was computed from

$$
B=\sum_{k} f_{. . k}\left(\bar{s}_{k}-\bar{s}\right)^{2}
$$

where $\bar{s}_{\mathbf{k}}$ is the mean of all inner square sizes in response class $R_{k}, f_{. k}$ is the frequency of use of response class $R_{k}$, and $\bar{s}$ is the mean of all inner square sizes over all 270 trials. The within-responseclass sum of squares, W, was computed from

$$
W=\sum_{k} \sum_{i} f_{i, k}\left(s_{i}-\overline{s_{k}}\right)^{2}
$$

The same measures were obtained from the Orthogonal conditions by collapsing the 3 by 3 response class tables to obtain a single column of cells in each re- sponse class identical to those of the Absolute and Constant conditions. The $\mathrm{B} / \mathrm{W}$ ratios for all conditions and all $O s$ are shown in Fig. 2. Although Os varied in the accuracy of classifying the sizes of the inner squares in the Absolute condition (indicated by the horizontal line) and in the gains in accuracy achieved under the Constant condition (zero range of variation of the outer square) the trend of results is the same for all. As outer square variation increased in range, accuracy in classing the size of the Inner square decreased to a level below that achieved under the Absolute condition.

This result appears to indicate that the general perceiver mode of operation was relatively stable under all conditions, the Os showing good performance when a relatively stable standard of judgment, the outer square, was avallable in the stimulus and poor performance when this standard became quite variable. The trend suggests that Os did not ignore this external standard even when it became an unreliable reference for judgment.

The horizontal line representing the result for the Absolute condition is crossed by the decreasing trend of results for the other conditions in about the same place for all $\mathrm{Os}$, suggesting a relatively constant relationship between accuracy obtainable under the Absolute condition and the critical range of variation of the outer square that produced the same accuracy. It is tempting to interpret the cross-over point as indicating the stability of the internal standard of Judgment used by $\mathrm{Os}_{\mathrm{s}}$ in the Absolute condition where no explicit external standard existed in stimulation. The remainder of the analysis indicates, however, that this is too simple an interpretation.

\section{The Dimensionality of the Response Classes}

The first test of the adequacy of the non-discriminating perceiver model consisted of the derivation of the discriminants in the stimulus space. The 1mplications of this extreme model are that the between-
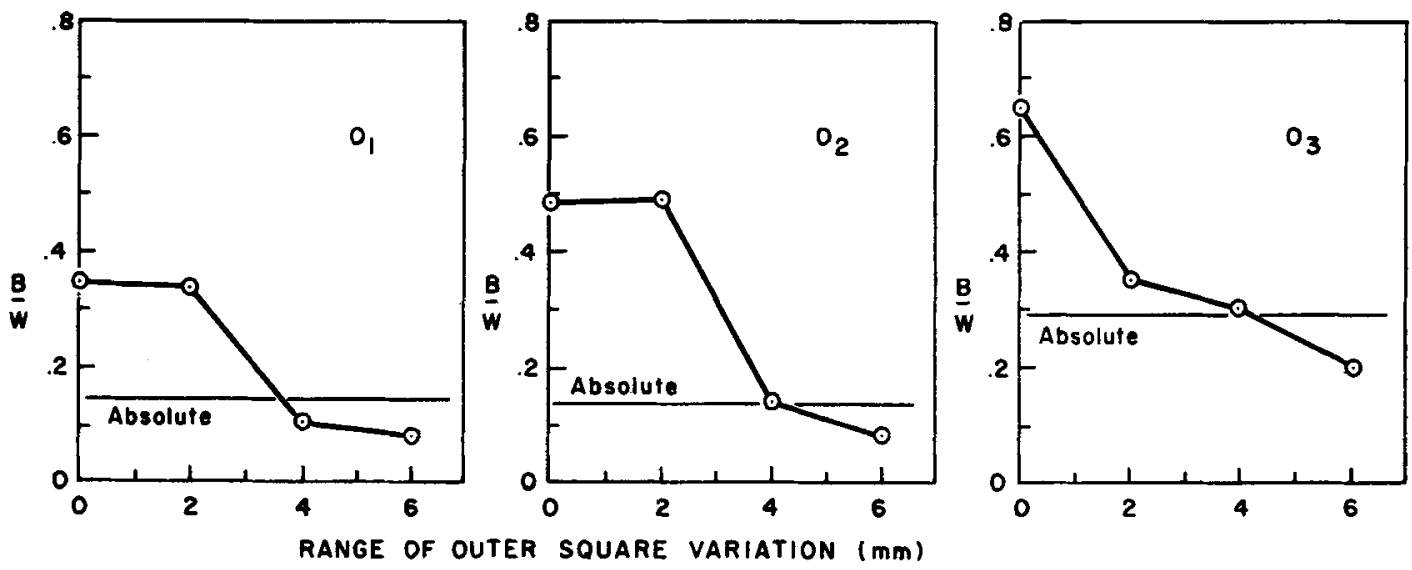

Fig. 2. Accuracy of identification of inner square size as measured by the ratio, $B / W$, or between to within response class sums of squares. Accuracy of Absolute judgment (inner square appearing alone) is indicated by the horizontal line for each 0 . 
response-class variation in each Orthogonal condition should be accounted for sufficlently by a single discriminant and that the ortentation of the single discriminant should be determined by the range of variation of the outer square sizes. As indicated earlier, these implications arise from the supposition that Os cannot discriminate at all between the perceptual effects of inner and outer square variation and respond instead to a univariate combination of the two.

The discriminant for each Orthogonal condition was derived by solving for $\lambda$ in

$$
\operatorname{det}\left[W^{-1} B-\lambda I\right]=0
$$

where $\mathrm{w}^{-1}$ is the inverse of the within sum of squares and cross-products matrix having as elements

$$
\begin{gathered}
w_{11}=\sum_{k} \sum_{i} f_{i . k}\left(s_{i}-\bar{s}_{k}\right)^{2} \\
w_{22}=\sum_{k} \sum_{j} f_{. j k}\left(S_{j}-\bar{S}_{k}\right)^{2} \\
w_{12}=w_{21}=\sum_{k} \sum_{i j} f_{i j k}\left(s_{i}-\bar{s}_{k}\right)\left(s_{j}-\bar{S}_{k}\right)
\end{gathered}
$$

and $B$ is the between sum of squares and crossproducts matrix having as elements

$$
\begin{aligned}
b_{11} & =\sum_{k} f_{. . k}\left(\bar{s}_{k}-\bar{s}\right)^{2} \\
b_{22} & =\sum_{k} f_{. . k}\left(\bar{s}_{k}-\bar{s}\right)^{2} \\
b_{12}=b_{21} & =\sum_{k} f_{. . k}\left(\bar{s}_{k}-\bar{s}\right)\left(\bar{S}_{k}-\bar{S}\right)
\end{aligned}
$$

in which $\bar{S}_{\mathrm{k}}$ refers to the average outer square size in response class $R_{k}$ and $\bar{S}$ refers to the mean of all outer square sizes in a condition. These elements were computed from 3 by 3 response tables like those in Table 2.

Two solutions for $\lambda$ are obtained in each condition. The first solution, $\lambda_{1}$, is the maximum value of the ratio

$$
\lambda_{1}=\frac{x_{1}^{\prime} B x_{1}}{x_{1}^{\prime} W x_{1}}
$$

in which $x_{1}$ is the vector of cosine numbers of a direction in the stimulus space. If the stimuli and the response centroids are projected on a line described by $x_{1}$, then the ratio of the between-response-class sum of squares to the within-response-class sum of squares evaluated along this line is maximized (Cooley \& Lohnes, 1962, Pp. 116-188). The cosine numbers in $\mathrm{x}_{1}$ are obtained by normalizing the solutions to

$$
\left(W^{-1} B-\lambda_{1} I\right) X_{1}=\left[\begin{array}{l}
0 \\
0
\end{array}\right]
$$

The second solution for $\lambda$ can be used to describe a second discriminant, orthogonal to the first, which may also account for some of the variation among response class centroids if they are not arranged in a line in the stimulus space. The proportion of the variation among response class centroids accounted for by the first discriminant alone is given by $\lambda_{1} /$ $\left(\lambda_{1}+\lambda_{2}\right)$. This is used here as a measure of the dimensionality of the response class centroids. The values of $\lambda_{1}, \lambda_{1} /\left(\lambda_{1}+\lambda_{2}\right)$, and $x_{1}$ are given in Table 3 for each $O$ and each of the Orthogonal conditions. The measure, $\lambda_{1}$, has the same meaning as the $\mathrm{B} / \mathrm{W}$ measures computed earlier for the classification of inner square size alone in the Orthogonal conditions. Those $\mathrm{B} / \mathrm{W}$ measures of accuracy of classification can, in fact, be obtained from the ratio, $\frac{X^{\prime} B X}{X^{\prime} W X}$, in which $B$ and $W$ are the sums of squares and crossproducts matrices obtained from (4) and (5) and $X$ is the vector $\left\{\begin{array}{ll}1 & 0\end{array}\right\}$. The $\lambda$ measure, then, is a general measure of the separation of the response class centroids along any single direction in the stimulus space, the direction being indicated by the vector $x$.

The very large values of $\lambda_{1} /\left(\lambda_{1}+\lambda_{2}\right)$ provide one answer to the question of the adequacy of the nondiscriminating perceiver model. It is obvious that in every case a single discriminant adequately accounts for the locations of the response class centroids in

\begin{tabular}{|c|c|c|c|c|c|c|c|c|c|}
\hline \multirow[b]{3}{*}{ Observer } & \multicolumn{9}{|c|}{ Range of Variation of the Outer Square } \\
\hline & \multicolumn{3}{|c|}{$2 \mathrm{~mm}$. } & \multicolumn{3}{|c|}{$4 \mathrm{~mm}}$. & \multicolumn{3}{|c|}{$6 \mathrm{~mm}}$. \\
\hline & $\lambda_{1}$ & $\frac{\lambda_{1}}{\lambda_{1}+\lambda_{2}}$ & $x_{1}$ & $\lambda_{1}$ & $\frac{\lambda_{i}}{\lambda_{1}+\lambda_{2}}$ & $x_{1}$ & $\lambda_{1}$ & $\frac{\lambda_{I}}{\lambda_{1}+\lambda_{2}}$ & $x_{1}$ \\
\hline$\underline{O}_{1}$ & .4606 & .9996 & {$\left[\begin{array}{r}.898 \\
-.439\end{array}\right]$} & .2496 & .9615 & {$\left[\begin{array}{r}.685 \\
-.728\end{array}\right]$} & .1056 & .9591 & {$\left[\begin{array}{r}.847 \\
-.532\end{array}\right]$} \\
\hline$\underline{\mathrm{O}}_{2}$ & .7306 & .9927 & {$\left[\begin{array}{r}.883 \\
-.469\end{array}\right]$} & 1.2946 & .9932 & {$\left[\begin{array}{r}.446 \\
-.895\end{array}\right]$} & 1.1812 & .9980 & {$\left[\begin{array}{r}.376 \\
-.927\end{array}\right]$} \\
\hline $\mathrm{O}_{3}$ & .5772 & .9740 & {$\left[\begin{array}{r}.847 \\
-.531\end{array}\right]$} & 1.1067 & .9859 & {$\left[\begin{array}{r}.665 \\
-.747\end{array}\right]$} & 1.3481 & .9931 & {$\left[\begin{array}{l}.528 \\
-.849\end{array}\right]$} \\
\hline
\end{tabular}
the stimulus space. That is, the response class centroids are scaled adequately on a single dimension, the appropriate single dimension in each condition being indicated by the vector of cosine numbers, $x_{1}$.

The $x_{1}$ of Table 3 indicate a simple linear combination of inner and outer square size. For $O_{1}$ in the 2 $\mathrm{mm}$ condition, for example, the indication is that the

Table 3. The Multiple Discriminant Analysis of the Orthogonal Conditions 
RANGE OF VARIATION OF OUTER SQUARE
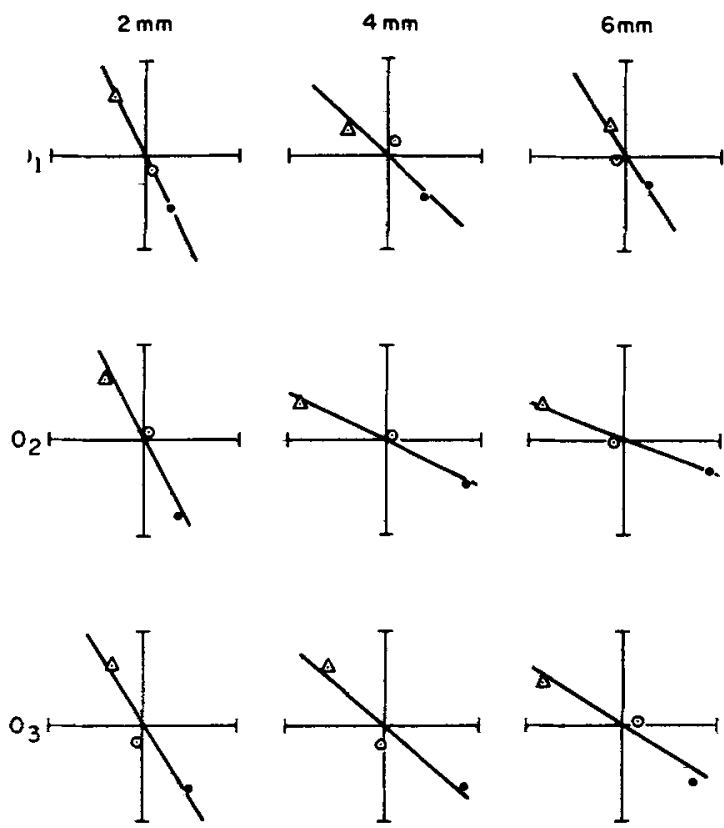

Fig. 3. The response class centroids and the discriminant for each $O$ in each of the Orthogonal conditions. The total length of each axis represents the total range of inner square (vertical axis) and outer square (horizontal axis) size variation measured in standard deviation units.

combination judged was $\left(.898 s_{i}-.439 s_{j}\right)$ where $s_{i}$ and $S_{j}$ are measured in units of their respective standard deviations. The $\mathrm{X}_{1}$ for $\mathrm{O}_{1}$ indicate that his discriminant was highly related to the vertical axis of inner square variation in all conditions. Separation of the response class centroids reflected on the discriminant, indicated by $\lambda_{1}$, decreased with increasing range of variation of the outer square. This $O$, then, does not represent the case predicted by the non-discriminating model. His discriminants do not show decreasing slopes as range of variation of the outer square increases, and the separation of his response class centroids does not increase with increasing range of variation of the outer square. Judged by these same criteria the remaining two Os do appear to have performed more like non-discriminating perceivers. Their performance is consistent with the supposition that in all Orthogonal conditions they judged only a linear combination of inner and outer square size, a combination that was increasingly determined by outer square size alone as the range of variation of that variable increased.

The discriminants derived for each $O$ in each Orthogonal condition are shown in Fig. 3. In each condition the discriminant is represented by a line drawn through the intersect of the inner square size (vertical) and outer square size (horizontal) axes.
The response centroids are also shown and are well fitted by the discriminant lines. The trends indicated in Table 3 are shown also in the figure in terms of the slope of the discriminant and separation of the centroids in each condition.

\section{The Prediction of Responses to Each Stimulus}

Further tests of the implications of the non-discriminating perceiver model were provided by an application of Bayes theorem previously used in the prediction of group membership in traditional measurement applications of multiple discriminant analysis (Cooley \& Lohnes, 1962, Pp. 134-138). The objective of the tests was to determine the extent to which the responses of each $O$ to each stimulus in each condition could be predicted on the basis of either of two alternative hypotheses. The first hypothesis was that each stimulus was classified by $O$ entirely in terms of its reflected location on the discriminant, i.e., $O$ was a non-discriminating perceiver. The second hypothesis was that each stimulus was classified instead by $O$ in terms of its two-dimensional location in the stimulus plane.

The adequacy of these alternative hypotheses about the dimensionality of the stimuli, as perceived by each $O$, was tested by the application of a prediction model involving some assumptions about the nature of the judgmental process on each trial in all conditions.

(1) When a stimulus, $s_{j} S_{j}$, was presented on a trial, it evoked a functional stimulus $s_{i}^{\prime} s_{j}^{\prime}$. Although $s_{j} S_{j}$ represents a stable, discrete point in the stimulus plane, $s_{j}^{\prime} s_{j}^{\prime}$ is considered to be a sample from a normal distribution defined over the stimulus plane. One such distribution is defined for each stimulus, and the centroid of the $s_{i}^{\prime} s_{j}^{\prime}$ evoked over many trials by any one $s_{j} S_{j}$ is assumed to be $s_{i} s_{j}$ itself.

(2) On any trial the judgment of $O$ was determined by the $s_{i}^{\prime} s_{j}^{\prime}$ evoked. He assigned $s_{i}^{\prime} s_{j}^{\prime}$ to the response class, $R_{k}$, for which the normalized deviation of $s_{j}^{\prime} s_{j}^{\prime}$ from the response class centroid, $\overline{\mathrm{s}}_{\mathrm{k}} \overline{\mathrm{S}}_{\mathrm{k}}$ was shortest.

In the case of the first hypothesis, that $O$ is a non-discriminating perceiver, each response class, $\mathbf{R}_{k}$, may be represented as a univariate normal distribution of all possible deviations $\left(s_{i}^{\prime} S_{j}^{\prime}-\bar{s}_{k} \bar{S}_{k}\right)$ measured along the discriminant in standard deviation units. Both $s_{i}^{\prime} s_{j}^{\prime}$ and $\bar{s}_{k} \bar{S}_{k}$ refer to reflected positions on the discriminant. Since the analysis yielding the discriminant assumes homogeneous response class variances, the standard deviations of all response classes measured along the discriminant are assumed equal and estimated by $\sqrt{\mathrm{X}_{1}^{\prime} \mathrm{DX}}$, in which $\mathrm{D}$ is the pooled within-response-class variance-covariance matrix, $D=W /(f . . .-3)$.

Assumption (2), then, is that $O$ classes the stim- 
ulus presented on each trial in the response class for which $Z_{k}$ is shortest, where

$$
z_{k}=\frac{\left(s_{i}^{\prime} s_{j}^{\prime}-\bar{s}_{k} \bar{S}_{k}\right)}{\sqrt{x_{1}^{\prime} \mathrm{Dx}_{1}}}
$$

and $s_{i}^{\prime} s_{j}^{\prime}$ is the functional stimulus evoked on that trial. Since there are three response classes in the experiment, three deviations were compared by $O$ on each trial.

Given these assumptions, the task of predicting the relative frequency with which stimulus $s_{1} S_{j}$ should be assigned to one response, $R_{1}$, is a simple one. It consists of computing the expected relative frequency with which deviations of the evoked $s_{j} s_{j}^{\prime}$ from the centroid $\bar{s}_{1} \bar{s}_{1}$ are shorter than their deviations from the other two centrotds. Since $s_{j} s_{j}$ is assumed to be the mean of all the $s_{i} s_{j}^{4}$ evoked by it, the expected relative frequency of assigning $s_{1} S_{j}$ to response class $R_{1}$, is given by

$$
\begin{gathered}
P\left(R_{1} \mid s_{i} S_{j}\right)=\frac{P\left(R_{1}\right) P\left(s_{i} s_{j} \mid R_{1}\right)}{\sum_{k} P\left(R_{k}\right) P\left(s_{i} s_{j} \mid R_{k}\right)} \\
k=1,2, \text { or } 3
\end{gathered}
$$

in which $P\left(R_{k}\right)$ refers to the relative frequency of usage of the response $R_{k}$ in a condition and $P\left(s_{j} S_{j} \mid R_{k}\right)$ is the ordinate of the normal curve at the deviation

$$
z_{k}=\frac{\left(s_{i} S_{j}-\bar{s}_{k} \bar{S}_{k}\right)}{\sqrt{x_{1}^{\prime} D X_{1}}}
$$

measured along the discriminant. Equation (9) specifies the likelihood that when $s_{i} S_{j}$ is presented the deviation of the evoked functional stimulus from $\bar{s}_{1} \bar{S}_{1}$ is the shortest and, hence, that $s_{1} S_{j}$ is assigned to response class $R_{1}$.

The reflected positions of the stimuli for each condition were obtained by premultiplying the 2 by 9 matrix of stimulus coordinates in the stimulus plane by the appropriate vector of cosine numbers, $x_{1}^{\prime}$. The reflected positions of the response class centroids were obtained from the premultiplication of the 2 by 3 matrix of centroid coordinates in the stimulus plane by the same appropriate vectors. Then the $\mathrm{Z}_{\mathrm{k}}$ were computed by means of Eq. (10), and these were used in providing the values of $P\left(s_{j} S_{j} \mid R_{k}\right)$ in Eq. (9).

The predicted relative frequencies, $P\left(R_{1} \mid s_{1} S_{j}\right), P\left(R_{2} \mid\right.$ $\left.s_{j} S_{j}\right)$, and $P\left(R_{3} \mid s_{i} S_{j}\right)$ were computed for the nine stimull in each condition for each $O$. Each of these relative frequencies was multipled by 30 (the number of appearances of each stimulus in each condition) to obtain the predicted frequencies. Only the "small" $\left(\mathbf{R}_{1}\right)$ and "large" ( $\left.\mathbf{R}_{3}\right)$ predicted frequencles were considered further since predictions of two responses determine the third completely.

The obtained frequencies of $R_{1}$ and $R_{3}$ are shown
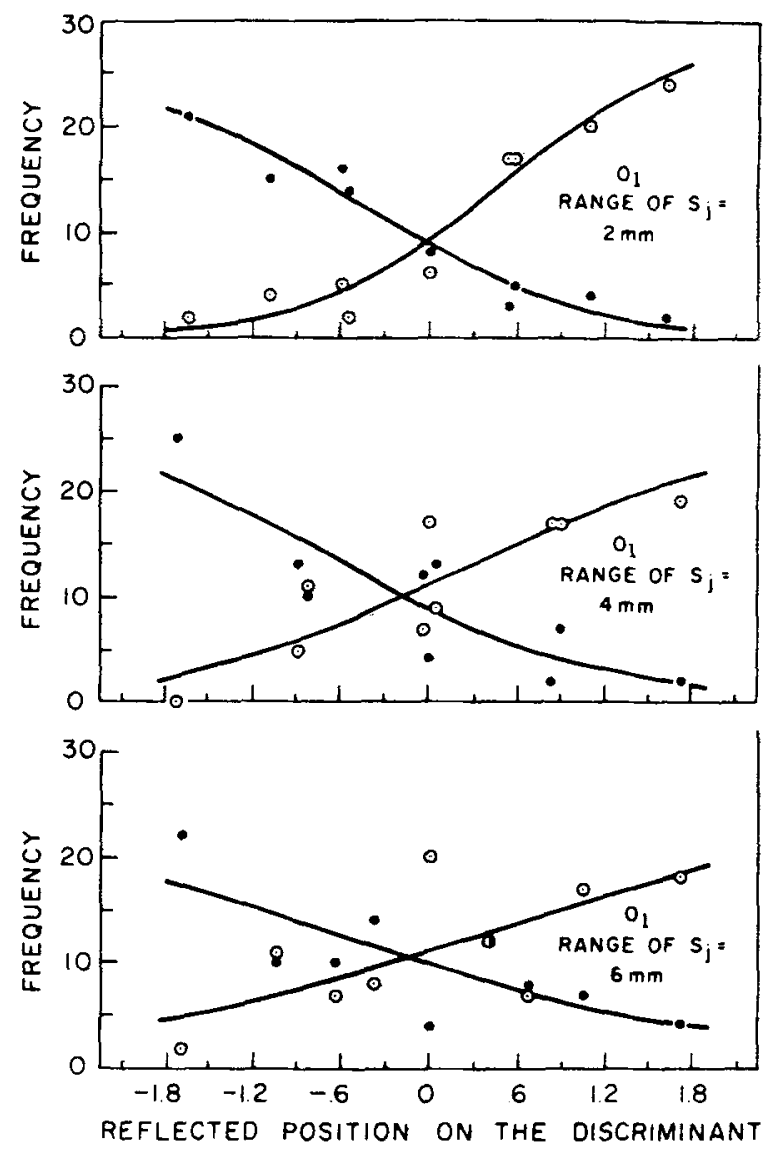

Fig. 4. Frequencies of "small" (closed circles) and "large" (open circles) responses to each stimulus are shown as a function of its reflected position on the discriminant. The lines are the predicted frequencies for all positions on the discriminant. Predictions assume the stimuli were effectively univariate. Data and predictions are for $\mathrm{O}_{1}$ and all Orthogonal conditions.

for the three Orthogonal conditions for $\mathrm{O}_{1}$ in Fig. 4, for $\mathrm{O}_{2}$ in Fig. 5, and for $\mathrm{O}_{3}$ in Fig. 6. These frequencies are plotted as a function of the reflected positions of the stimuli on the discriminant appropriate for each condition. Negative values on the discriminant represent reflections on the lower right-hand portion of a discriminant, as plotted in Fig. 1. Positive values represent positions on the upper left-hand portion. Predicted frequencies are corresponding points on the continuous functions, which provide predicted frequencies for all points on the discriminants.

The figures indicate considerable precision in accounting for the obtained frequencies under the assumption that the stimuli, as perceived by $O$, can be adequately scaled as points on a line rather than as points in a plane. An indication of this precision is shown in Table 4. Column 3 contains the pooled variances within the "small" and "large" response classes for each of the Orthogonal conditions and each $O$. Column 4 indicates the proportion of each pooled variance accounted for by the correlation 

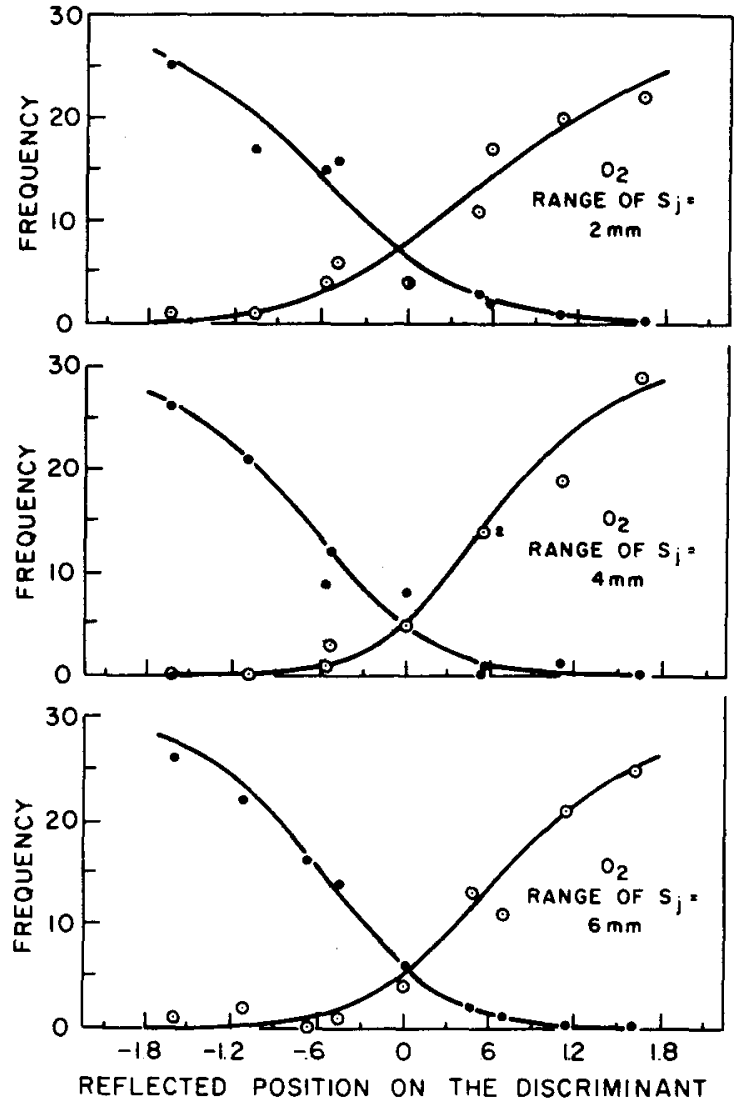

Fig. 5. The predicted and obtained frequencies for $\mathrm{O}_{2}$.

between obtained and predicted frequencies within the two response classes. For $\mathrm{O}_{2}$ and $\mathrm{O}_{3}$ these proportions are large and increase as the range of var1ation of the outer square increases. Hence, both appear to conform well to the non-discriminating perceiver model. The result for $O_{1}$ indicates that a univariate representation of the stimuli is adequate only for the $2 \mathrm{~mm}$ condition and becomes more and more inadequate for the other conditions.

Column 5 of Table 4 indicates the proportions of each pooled variance accounted for by the other hypothe-
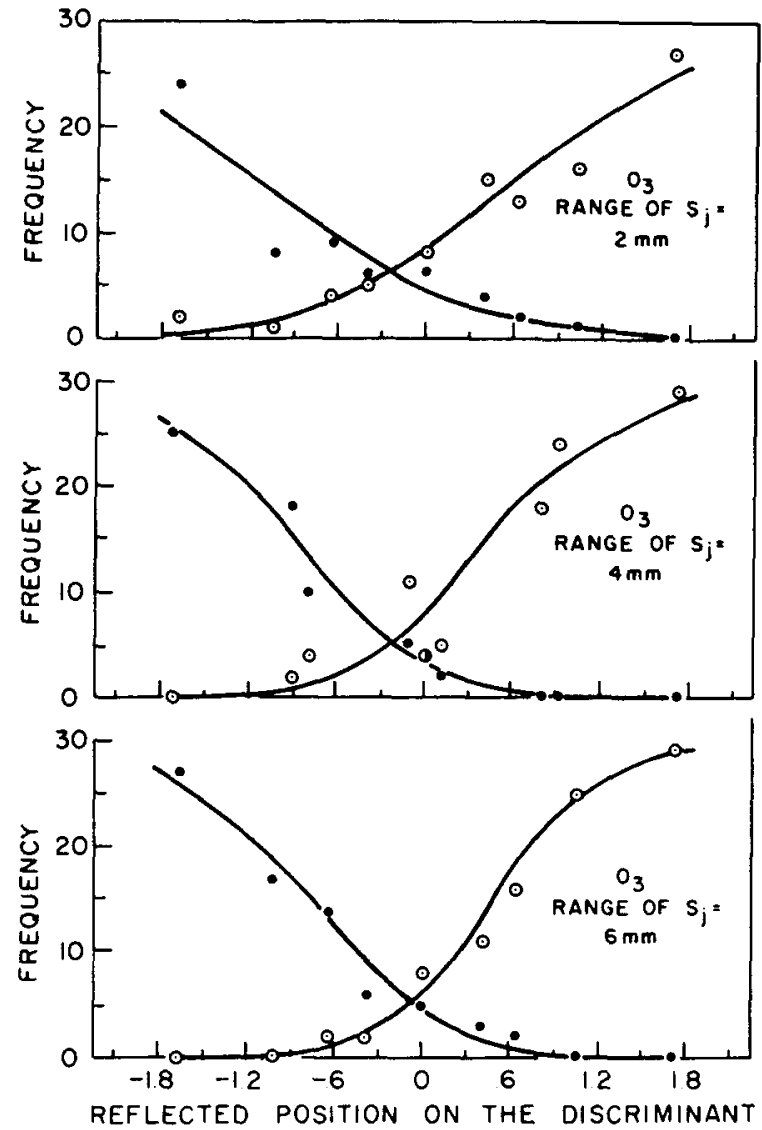

Fig. 6. The predicted and obtained frequencies for $\mathrm{O}_{3}$.

sis, that each stimulus was classified by the Os in terms of its two-dimensional location in the stimulus plane. These proportions were obtained by correlating the obtained frequencies in each condition with a set of predicted frequencies obtained by another predictive equation. This new equation was derived from the same assumptions underlying Eq. (9) but assumes also that the deviations, $Z_{k}$, which $O$ compares on each trial are measured in the direction of $s_{i}^{\prime} s_{j}^{\prime}$ from $\overline{\mathbf{s}}_{\mathbf{k}} \overline{\mathbf{S}}_{\mathbf{k}}$ in the stimulus plane. That is, the functional stimulus, $s_{j}^{\prime} S_{j}^{\prime}$, evoked by $s_{j} S_{j}$ is a sample from a

Table 4. Response Class Variances and Proportions Accounted for by Predictions of Two Perceiver Models.

\begin{tabular}{|c|c|c|c|c|c|}
\hline Observer & $\begin{array}{c}\text { Orthogonal } \\
\text { Condition }\end{array}$ & $\begin{array}{l}\text { Response-Class } \\
\text { Variance }\end{array}$ & $\begin{array}{l}\text { Proportion Predicted } \\
\text { from Discriminant }\end{array}$ & $\begin{array}{l}\text { Proportion Predicted by } \\
\text { Two-dimensional Stimulus } \\
\text { Locations }\end{array}$ & $\begin{array}{c}\text { Proportion Predicted } \\
\text { within } \\
\text { Outer Square Sizes }\end{array}$ \\
\hline$\underline{O}_{1}$ & $\begin{array}{l}2 \mathrm{~mm} \\
4 \\
6\end{array}$ & $\begin{array}{l}57.27 \\
45.79 \\
31.98\end{array}$ & $\begin{array}{l}.9430 \\
.7389 \\
.5268\end{array}$ & $\begin{array}{l}.9476 \\
.8256 \\
.6638\end{array}$ & $\begin{array}{l}.9675 \\
.9596 \\
.8697\end{array}$ \\
\hline $\mathrm{O}_{2}$ & $\begin{array}{l}2 \mathrm{~mm} \\
4 \\
6\end{array}$ & $\begin{array}{l}70.72 \\
91.82 \\
89.32\end{array}$ & $\begin{array}{l}.9421 \\
.9694 \\
.9773\end{array}$ & $\begin{array}{l}.9522 \\
.9828 \\
.9828\end{array}$ & $\begin{array}{l}.9908 \\
.9757 \\
.9956\end{array}$ \\
\hline$\underline{\mathrm{O}}_{3}$ & $\begin{array}{l}2 \mathrm{~mm} \\
4 \\
6\end{array}$ & $\begin{array}{l}60.96 \\
92.76 \\
97.27\end{array}$ & $\begin{array}{l}.9088 \\
.9351 \\
.9775\end{array}$ & $\begin{array}{l}.9414 \\
.9563 \\
.9912\end{array}$ & $\begin{array}{l}.9748 \\
.9797 \\
.9966\end{array}$ \\
\hline
\end{tabular}


bivariate normal distribution defined over the stimulus plane; and the normalized deviation of $s_{i}^{\prime} s_{j}^{\prime}$ from $\overline{\mathrm{s}}_{\mathrm{k}} \overline{\mathrm{S}}_{\mathrm{k}}$ is given by

$$
\begin{gathered}
z_{k}=\left[\begin{array}{ll}
\left(s_{i}^{\prime}-\bar{s}_{k}\right) & \left(S_{j}^{\prime}-\bar{S}_{k}\right)
\end{array}\right] \\
z_{k}=\sqrt{z_{k}^{\prime} D_{k}^{-1} z_{k}}
\end{gathered}
$$

in which $D_{k}^{-1}$ is the inverse of the within-responseclass variance-covariance matrix for response class $R_{k}$ alone. The deviation $Z_{k}$, then, measures the deviation of $s_{i}^{\prime} s_{j}^{\prime}$ from the centroid $\bar{s}_{k} \bar{s}_{k}$, in the direction of $s_{j}^{\prime} S_{j}^{\prime}$ from the centroid and in units of the response class standard deviation evaluated in that direction.

Since $s_{j} S_{j}$ is again assumed to be the centroid of all the $s_{i}^{\prime} S_{j}^{\prime}$ evoked by it, the expected relative frequency of assigning $s_{i} S_{j}$ to response class $R_{1}$ is given by

$$
\begin{aligned}
P\left(R_{1} \mid s_{i} S_{j}\right) & =\frac{\frac{P\left(R_{1}\right)}{\left|D_{1}\right|^{1 / 2}} e^{-Z_{1}^{2} / 2}}{\sum_{k} \frac{P\left(R_{k}\right)}{\left|D_{k}\right|^{1 / 2}} e^{-Z_{k / 2}^{2}}} \\
k=1,2, \text { or } 3 &
\end{aligned}
$$

Again, the quantities $P\left(R_{1} \mid s_{1} S_{j}\right)$ and $P\left(R_{3} \mid s_{i} S_{j}\right)$ were computed for each of the nine stimuli in each condition, multiplied by 30 to obtain the predicted frequencies, and correlated with the obtained frequencies. The squares of these correlations are presented in Column 5 of Table 4 .

An increase in the precision of prediction is indicated in all cases, although the increases for $\mathrm{O}_{2}$ and $\mathrm{O}_{3}$ are quite small. Their judgments are already accounted for quite well by unidimensional representations of the stimuli. The increases for $O_{1}$, however, indicate that in the $4 \mathrm{~mm}$ and $6 \mathrm{~mm}$ conditions a two-dimensional representation of the stimuli results in a considerable gain in the precision of prediction. This again indicates that his performance does not conform well to the non-discriminating perceiver model when variation of the outer square size is either $4 \mathrm{~mm}$ or $6 \mathrm{~mm}$ in range.

The last column of Table 4 indicates the maximum predictive precision possible, given the assumptions used in describing the judgmental process in each trial. Both Eq. (9) and Eq. (12) involve parameters obtained from the data of a single condition. In the case of Eq. (9) these are the $P\left(R_{k}\right)$, the relative frequency of use of each response; $[\mathrm{D}]$, the pooled within-response-class variance-covariance matrix; the three $\bar{s}_{k} \bar{S}_{k}$, the response class centrotds; and $X$, the vector of cosine numbers. With a specified, limited number of trials these are not all independent aspects of the data. Only two of the $P\left(R_{k}\right)$ and two of the $\overline{\mathbf{s}}_{\mathrm{k}} \overline{\mathrm{S}}_{\mathrm{k}}$ are independent. The vector $\mathrm{X}$ is determined by the $P\left(R_{k}\right)$, the $\bar{s}_{k} \bar{S}_{k}$, and [D]. Increases in the number of stimuli do not increase the number of parameters involved in prediction. Hence, a prediction for any reflected position on the discriminant involves the same set of five independent parameters.

Predictions computed from the use of Eq. (12) require the use of $\left[D_{k}\right]$ rather than [D]. This adds one more independent data-derived parameter, since only two of the $\left[D_{k}\right]$ are independent if $[D]$ is specified. Again, the number of parameters required is not increased by increases in the number of stimuli used.

We wished to compare the predictive accuracy obtainable from the use of the limited set of data-derived parameters involved in Eqs. (9) and (12) with the case producing the maximum possible predictive accuracy permitted by the assumptions. This was produced by making separate predictions for the three inner square sizes within each outer square size class.

Response class centroids were computed on the basis of the judgments of the three stimuli in each column of Fig. 1. This produced three centroids for the first column $\left(S_{j}=-Z_{j}\right)$, three centroids for the second column $\left(S_{j}=0\right)$, and three for the third column $\left(S_{j}=+Z_{j}\right)$. The three centrolds for the first column, the pooled response class standard deviation for that column, and the inner square sizes for that column provided the values for Eq. (9). This was repeated for the second and third columns of Fig. 1.

The predicted frequencies obtained in this way involve the maximum number of data-derived parameters and, hence, test the limit of predictive accuracy possible under the assumptions made about the judgmental process. They provide a ceiling against which the accuracies of prediction produced under the two hypotheses can be contrasted. It is apparent that this ceiling is quite high in all cases. This provides support for the basic judgmental model underlying the predictive equations used in evaluating the two hypotheses concerning modes of perceiver operation. It also indicates, in the case of $O_{1}$, that his judgments of the stimuli are accurately predictable in the judgmental system assumed. Hence, the lack of precision permitted by the use of Eqs. (9) and (12) for $O_{1}$, relative to that possible for the outer two $O s$, indicates a fallure of the hypotheses underlying those equations rather than an inability to predict his responses under any hypothesis. An adequate accounting of his judgments requires the hypothesis that when outer square variation was large $O_{1}$ judged inner square sizes uniquely within outer square size classes. This hypothesis was not rigorously tested by the data, of course, because of the large number of data-derived parameters involved relative to the number of stimuli for which predictions were produced.

\section{Discussion}

A basic supposition underlying this experimentation was that a mode of perceiver operation producing performance in the Constant condition superior to 
that in the Absolute condition, where no outer square was present on a trial, would imply a particular result in the Orthogonal conditions. That is, superior performance in the Constant condition would indicate reliance on outer square size as a judgmental standard; and this reliance should produce poor performance in judging inner square size when outer square size varied also, provided the mode of operation of the Os was constant over the conditions.

The results shown in Fig. 2 confirmed this supposition. All Os performed best in the Constant condition, and the performance of all deteriorated progressively with increasing range of outer square size in the Orthogonal conditions. None appeared to suppress a reliance on outer square size as a judgmental standard in the noisy Orthogonal conditions where outer square size provided an unreliable basis for judgment of inner square size. The mode of operation of all involved a reliance on outer square size, and this mode must have been relatively constant over all the conditions.

These results, as shown in Fig. 2, are emphasized because they provide an interesting contrast with the information yielded by the discriminant analysis. In the latter case a marked difference in the performance of $\mathrm{O}_{1}$, as opposed to that of $\mathrm{O}_{2}$ and $\mathrm{O}_{3}$, was clearly demonstrated.

The performances of $\mathrm{O}_{2}$ and $\mathrm{O}_{3}$ were accounted for adequately by the non-discriminating perceiver model, i.e.s by the assumption that the effective stimuli judged were simply points on the discriminant derived in each condition. In terms of their ability to judge the variable represented by the discriminant, their performance became better and better as outer square size variation increased. However, the variable they judged, when outer square size variation was considerable, was not strongly related to inner square size variation.

A different type of performance was demonstrated by $O_{1}$, who appears to have made an attempt to partial out or suppress outer square size variation as a stimulus in the Orthogonal conditions. The mode of operation employed may have been an attempt to class the stimulus combinations in terms of outer square size classes and to judge inner square size within these classes. This produced discriminants that were more highly related to inner square size alone. It did not, however, produce more reliable judgment of inner square size, as Fig. 2 clearly shows. Nor did it produce reliable judgment of the type of stimulus varlation represented by the discriminants, as the small values of $\lambda_{1}$, in Table 3 clearly show.

These results, those of Fig. 2 and the information provided by the discriminant analysis, indicate that the questions of how much and how constantiy a contextual stimulus aspect, such as the outer square variation, affects judgment of a critical aspect of stimulation, such as the inner square variation, may be too simple. The resuits suggest that the $O$ has options. He may, as did $\mathrm{O}_{2}$ and $\mathrm{O}_{3}$, respond to a dorninant mode of stimulus variation apparent from trial to trial, a mode of variation that combines critical and contextual stimulus aspects. When contextual variation is considerable, as in the $4 \mathrm{~mm}$ and $6 \mathrm{~mm}$ conditions, this gives $\mathrm{O}$ a satisfying variable to judge. His apparent consistency of judgment can be quite high. OI he may, as did $O_{1}$, attempt to partial out the aspect of stimulus variation that appears to be contextual rather than critical stimulus variation. This distinction may be restated as the distinction between an attempt to satisfy a coherence criterion of judgment (Hake et al, 1966; Rodwan \& Hake, 1964), which leads to maximum subjective response consistency, and an attempt to satisfy an accuracy or veridical criterion.

If Os have these options under judgmental conditions represented by the Orthogonal conditions of this experiment, then a further conclusion must be drawn. That is that the same limitation upon the accuracy with which critical stimulus variation is judged exists for either option. This limitation can be depicted in terms of the stimulus space of Fig. 1. The suggestion is that stimulus variation, represented by movement of a point in this space, is not equally resolvable in all directions. Movement in a direction parallel with the discriminant may be most highly resolvable; movement in any other direction being less so. Hence, the option is between responding to the more resolvable variation represented by the discriminant, variation that is only partially related to inner square size (the vertical axis), and responding to less resolvable variation that is more nearly colinear with inner square size variation.

The data of this experiment, as analyzed, support this depiction of the options available to the Os. While these options may satisfy different subjective criteria for $O s$, the data indicate they are equivalent in terms of ability to reliably identify inner square size alone.

\section{References}

Cooley, W. W.. \& Lohnes, P. R. Multivariate procedures for the behavior sciences. New York: Wiley, 1962.

Hake, H. W., Rodwan, A., \& Weintraub. D. Noise teduction in perception. In K. R. Hammond (Ed.), The psychology of Egon Brumswick. New York: Holt, Rinehart \& Winston, 1966. Pp. $277-$ 316.

Künnapas, T. M. Influence of frame size on apparent length of a linie. J. exp. Psychol., 1955, 50, 168-170.

Obonai, $\mathbf{T}$. Induction effects in estimates of extent. J. exp. Psychol, 1954, 47, 57-60.

Rock, I., \& Ebentoltz, s. The relational determination of perceived size. Psychol. Rev., 1959, 66, 387-401.

Rodwan, A. S., \& Hake, H. W. The linear discriminant function as a model for perception. Amer. J. Psychol., 1964, 77, 380-392.

Uhr, L. "Pattern recognition" computers as models for form perception. Psychol, Bull, 1963, 60, 40-73.

\section{Note}

1. Supported in part by a grant from the National Science Foundation (GB-5280).

(Accepted for publication dune 19.1967.$)$ 